\title{
A study on Hope, Stress and Self-Efficacy in Parents of Children with Intellectual Disability
}

\author{
Rupa Kore ${ }^{1}$, Saindhavi Venkatraman ${ }^{2}$ \\ ${ }^{1}$ Post Graduate Student \\ ${ }^{2}$ Assistant Professor \\ Department of Psychology, Maniben Nanavati Women's College, Mumbai \\ E-mail-sayhi2rupa@gmail.com \\ Corresponding Author - Rupa Kore
}

\begin{abstract}
Introduction: The purpose of the study is to understand the impact of hope on perceived stress and selfefficacy among parents of children suffering from intellectual disability. Due to the researcher's personal experience as well as working with the parents or caregivers of children with intellectual disability has always been the researcher's area of interest. Another reason behind doing research in the area of intellectual disability is to study and understand how positive factors such as hope \& self-efficacy can help the parents \& family members to see the disability or the situation in a positive way and deal with it more effectively.

Methodology: 60 participants were purposively selected from resource rooms of special schools, hospitals and private intellectual disability centers. The head of the centres and the principals of the schools and parents were contacted to provide information regarding the objective of the research, scales, principle of confidentiality and to seek their permission for the study. The data was collected using the survey method. The General Perceived Self-Efficacy Scale (GPSS), Procrastination Scale and the Brief Resilience Scale will be administered on each of the students.

Results: The first hypothesis was parents of children with intellectual disability having higher level hope will score lower on perceived stress as compared to parents with lower level hope. The statistical result obtained was in line with the hypothesis and also was in line with previous studies. The second hypothesis was parents of children with intellectual disability having higher level hope will have higher level of selfefficacy as compared to parents with lower level hope. The statistical result found was in line with the second hypothesis and also in line with past studies.

Conclusion: The findings indicate that the parents having higher level hope had lower level of perceived stress and higher level of self-efficacy as compared to parents with lower level hope. The results obtained are in line with hypotheses and past research.
\end{abstract}

Key Words: Hope, self efficacy, stress, perceived stress, intellectual disability, parents.

(Paper received $-22^{\text {nd }}$ June 2017, Peer review completed $-18^{\text {th }}$ July 2017, Accepted $-20^{\text {th }}$ July 2017)

\section{INTRODUCTION}

Parents of children with intellectual disability experience deleteriously high levels of stress. Parenting stress is an anxiety and tension related to the role of a parent [1]. Perceived stress is defined as the degree to which situations in one's life are appraised as stressful [2]. Research on families of Children with intellectual disability has repeatedly indicated three stressful effects of the intellectual disability - a) Social isolation increased indicators of stress in the parents and c) a greater incidence of problems in school and in the society when the parents learn that the child is having some form of disability, it causes enormous distress to them [3]. Giving birth to a child with disability is a stressful event which affects the whole 
family. Such an event may impair family development and may continue over time affecting the entire family system. The impact of the disability and problem associated with it are not restricted to the child but extend far beyond the child and affects a number of areas of family functioning. Parents go through intense emotional and psychological stress and may have fewer resources of emotional gratification. They may consider mentally handicapped child as a threat to their self-esteem and view themselves as a source of disability [4-5]. Self-efficacy is explained in the theoretical framework of social cognitive theory by Bandura [6] which stated that human achievement depends on interactions between one's behaviors, personal factors and environmental conditions. The diagnosis of intellectual disability in a child triggers a range of emotional responses in parents. For some, it is a crisis that requires extraordinary psychological adjustment on a parent's part and contains elements of harm, loss and weakness. For others, the birth of a child with disability is viewed as an unfortunate event, yet one that has positive implications. Besides, child's problematic behaviour, parent's own physical and mental health is also at risk. The quality and quantity of impact may vary from parent to parent, depending upon the social, physical, and emotional support. However, some parents show better tolerance of these stressors than others. This may be due to the certain factors such as hope, self-efficacy, social support, sense of coherence, self-esteem, hardiness, optimism, and internal locus of control [7-8].

\section{Socio-cultural Factors}

Children with mental retardation are more likely to come from low socioeconomic backgrounds. This may be because their parents also have mental retardation and have not been able to acquire well-paying jobs. The social disadvantages of being poor also may contribute to lower than average intellectual development. Poor mothers are less likely to receive good prenatal care, increasing the risk of premature birth. Children living in low socioeconomic areas are at increased risk for exposure to lead, because many older buildings have lead paint, which chips off and can be ingested. Poor children are concentrated in poorly funded schools, where those with lower IQs receive less favourable attention from teachers and fewer learning opportunities, especially if they are also members of minorities. Poor children also are less likely to have parents who read to them and are involved in their schooling. These factors may directly affect a child's intellectual development and exacerbate the biological conditions that impede a child's cognitive development [9-10].

\section{Hope}

A study done explored the relationships among hope, optimism, social support and parenting behaviors as well as hope, optimism, stress and parenting behaviors while controlling for social support in a sample of caregivers of children with intellectual disabilities. It was hypothesized that hope and optimism will moderate the relationship between social support and parenting behaviors and that hope and optimism will moderate the relationship between stress and parenting behaviors when controlling for social support. Neither hope nor optimism moderated the relationship between social support and positive parenting behaviors. In the study social support and hope were found to predict positive parenting behaviors in caregivers of children with intellectual deficits. Optimism was not found to predict a significant amount of the variance in positive parenting behaviors. Neither hope nor optimism moderated the relationship between stress and negative behaviors when controlling for social support. Stress was found to predict positive but not negative parenting behaviors when controlling for social support [11].

Another study done in Pakistan aimed to explore the relationship between Depression, Hopelessness and Associated Attitude among parents of mentally retarded children. Correlation analysis was done and results yielded that there is highly significant relationship between hopelessness and attitude in parents of MR children whereas no significant relationship was found between depression and parental attitude. Research findings suggest that parents of mentally retarded children show less psychological symptoms which may be due to positive perception, parental strengths and resilience among them [12].

Another research done aimed at comparing Worry and hope between mothers having children with Autism syndrome and Down syndrome. The results showed that the amount of worry and hope among mothers having children suffering from autism is different from mothers having children suffering from Down syndrome. The Worry between mothers having children with autism syndrome was more than 
mothers having children with Down syndrome. The level of hope between mothers having children with Down syndrome was higher than mothers having children with autism syndrome [13].

A comparative cross-sectional study done tried to compare difference of mental and physical stress between the parents of children with mental retardation and the parents of children with no mental retardation. The study revealed that the parents of children with mental retardation shared significantly greater stress score than the parents of children with no mental retardation. Majority of the parents with mentally retarded children had higher mental stress than physical stress. Mothers had significantly higher mental stress score than the fathers of mentally retarded children [14].

Researchers identified the Predictors of parental stress and psychological distress among parents of children with mental retardation in the United Arab Emirates. The results indicate that the age of the child was significantly associated with parents' feelings of distress and psychiatric symptom status, and parental stress was less when the child was older. Fathers' work appeared to be a significant predictor of parental stress, indicating that for fathers who were not working the level of stress was higher than fathers who were working. Lower socio-economic level was associated with greater symptom rates of cognitive disturbance, depression, anxiety, and despair among parents [15].

Researchers did a study to determine the parenting stress and its determinants among parents of children with disabling conditions in India. Results found out that the female sex of the child was associated with higher stress related to failure of the child to meet parent's expectations and to satisfy the parents in their parenting role. Parents engaged in more lucrative and prestigious occupations had more stress than parents engaged in less prestigious and lucrative occupations irrespective of their income. Many parents reported receiving little support from their extended families in taking care of their child. Religion was found to be a common coping resource used by the parents [16].

Parents having children with Mental Retardation get disturbed with added responsibilities associated with care of their children in the normal day to day functions. This may exert pressure on the parents psychologically, physically and in their social functioning. It is necessary to look at the issues related to how parents of children with intellectual disability suffer from social problems and the ways of preventing and overcoming their problems in the society they live in [17].

Besides, child's problematic behavior, parents own physical and mental health is also at risk. The quality and quantity of impact may vary from parent to parent, depending upon the social, physical, and emotional support. Interaction as well as the relationship between parents with their family members and friends is also affected. Due to their child's disability, parents have to suffer an unending sense of loss. Their expectation about the child goes up sided down. This feeling of loss and expectation about their child can lead them to a long-term cyclical distress or sadness. However, some parents show better tolerance of these stressors than others. This may be due to the certain factors such as hope, self-efficacy, social support, sense of coherence, self-esteem, hardiness, optimism, and internal locus of control [18].

Research done examined the relationship of different types of family support to life satisfaction and parenting self-efficacy (PSE), and explored whether income and ethnicity moderated these relationships. Interviews with 84 Latino and 37 White participants revealed that partner emotional support predicted life satisfaction and PSE in both ethnic groups, with a stronger relationship evident for the PSE of Latino mothers. Income was not a significant moderator [19].

The research aims at finding that whether the levels of hope affect the perceived stress and self-efficacy of parents of children with intellectual disability. The research does not aim at providing an intervention in cases of observed issues. However, if there is any area of concern identified, the responsible person was informed so that they could take the necessary steps.

\section{METHODOLOGY}

\section{Hypotheses}

Hypothesis 1 - Parents having high hope will score lower on perceived stress as compared to the parents having low hope.

Hypothesis 2 - Parents having high hope will score higher on self-efficacy as compared to the parents having low hope. 


\section{Participants}

The sample consisted of 60 parents of children who were certified as having mild-moderate intellectual disability in the age range of 8 to 16 years. Participants of both genders were included 41 females and 19 males. The criteria for the research entailed that, all the children must be certified as having mild-moderate intellectual disability and the parents should have never been diagnosed with any psychiatric disorder or physical disability and they should not be single / divorced / widowed / separated as well as should be residing in Mumbai. The data was collected from resource room of special schools, hospitals and private intellectual disability centres. Non-probability sampling method was used and primary data was collected using purposive sampling. The centres were selected on the basis of their geographical proximity to the researcher. The researcher prepared a list of centres, in rank order, of accessibility. Once some centres agreed to participate in the study, negotiations with other centres ceased.

\section{Variables}

The research has One Independent Variable with Two categories of High Hope and Low Hope. The research has Two Dependent Variables- Perceived Stress and Self-efficacy.

\section{Instruments}

1. The Adult Trait Hope Scale (ATHS) [20].

2. The Perceived Stress Scale (PSS) [21].

3. The General Self-efficacy Scale (GSES) [22].

\section{Procedures}

Sixty participants were purposively selected from resource rooms of special schools, hospitals and private intellectual disability centres. The head of the centres and the principals of the schools and parents were contacted to provide information regarding the objective of the research, scales, principle of confidentiality and to seek their permission for the study. Once they agreed to allow the study to be carried out and the signed consent letters of the institute were acquired; the researcher arranged an appropriate date and time for administering the questionnaires. The data was collected using the survey method. The General Perceived Self-Efficacy Scale (GPSS), Procrastination Scale and the Brief Resilience Scale will be administered on each of the students. The parents who were willing to participate in the study were provided with standardized instructions before the administration. They were explained the purpose and relevance of the study. After that these parents completed the paper and pencil questionnaire. The researcher was present to answer any questions the parents had. After each participant was finished with the survey and the materials were collected, they were thanked for participating in the study. After the surveys were collected from the centres, data were scored, coded and entered into an excel spreadsheet. Data coding and entry were verified to minimize error. With the help of Microsoft Excel statistical analysis was done.

\section{STATISTICAL ANALYSIS}

The result of the collected data was quantitatively analyzed. There is one independent variable-Hope. The scores obtained on the Independent variable were divided using median split. The value for median split was 42.5. Hence there are two levels of the Independent Variable, i.e. high and low. The participants, who scored above 42.5, fall under the higher level of hope. Whereas the participants who scored lower than 42.5 were participants with lower level of hope. The inferential statistics used is independent t-test. The analysis of the data was carried out using Microsoft Excel. Mann Whitney U tests were administered to rule out gender differences among participants as the sample size of both the genders was not equal. 


\section{RESULTS}

Apart from the above-mentioned scores, the descriptive statistics of the dependent variable of perceived stress; revealed that the mean value for the participants who scored high on hope was 19.16 with a standard deviation of 6.38. Moreover, scores varied between 31 and 3 .

On the other hand; statistical results of perceived stress, on participants who scored a lower level of hope showed that the mean was 27.9 and standard deviation was 5.31. The scores obtained ranged between 37 and 17.

Table1: Descriptive statistics of the variables

\begin{tabular}{|c|c|c|c|}
\hline Variables & Range & Mean & S D \\
\hline Hope & $64-23$ & 42.62 & 10.50 \\
\hline Perceived Stress & $37-3$ & 22.06 & 7.30 \\
\hline Self-efficacy & $40-15$ & 27.04 & 6.72 \\
\hline
\end{tabular}

Apart from the above-mentioned scores, the descriptive statistics of the dependent variable of perceived stress; revealed that the mean value for the participants who scored high on hope was 19.16 with a standard deviation of 6.38. Moreover, scores varied between 31 and 3. On the other hand; statistical results of perceived stress, on participants who scored a lower level of hope showed that the mean was 27.9 and standard deviation was 5.31. The scores obtained ranged between 37 and 17.

The mean and standard deviation of participants scoring a high level of hope on self-efficacy was 32.4 and 4.90 respectively and the scores ranged from 40 to 23 . On the contrary; statistical results of self-efficacy, on participants who scored a lower level of hope showed that the mean was 22.8 and standard deviation was 4.09. The scores obtained ranged between 32 and 15 . Before performing t-test, normality and homogeneity were checked. For self-efficacy, skewness and kurtosis values are 0.093 and -0.96 respectively. These values remain in between the range of -1 and +1 , which is an acceptable range for the normality range for t-test.

Skewness and kurtosis values were examined on the participants to check the normality of dependent variables. The results revealed that for the high scorers of hope for perceived stress, the statistics was -0.198 for Skewness and 0.068 for Kurtosis. The skewness and kurtosis values for perceived stress of the participants who scored less on hope are -0.281 and -0.328 respectively. The value of Skewness and kurtosis for self-efficacy in participants with high hope level are -0.343 and -0.660 respectively, whereas for the lower level hope scorers the skewness and kurtosis values are 0.137 and -0.296 respectively. These values remain in between the range of -1 and +1 , which is an acceptable range for the normality range for $t$ test.

The obtained $t$ value for participants scoring a higher as well as lower level of hope is 5.758 . The degree of freedom for the higher and low level scorers on hope is 58 . For perceived stress it was observed that the level of significance for both the higher and the lower level of hope participants was 0.00001. Since the obtained value is less than 0.05 level of significance; the data is calculated is found to be significant. The first hypothesis tested whether parents of children with intellectual disability having higher level hope will score lower on perceived stress as compared to parents with lower level hope. In lieu of the above results, the first hypothesis was verified. Hence, null hypothesis can be rejected, and hypothesis 1 can be accepted.

The $t$ value for participants scoring a higher as well as lower level of hope is -8.229 . The degree of freedom for the higher and lower level scorers on hope is 58. For self-efficacy it was observed that the level of significance for both the higher and the lower level of self-efficacy participants was 0.00001 . Since the obtained value is less than 0.05 level of significance; the data is calculated is significant. The second hypothesis tested whether parents of children with intellectual disability having higher level hope will have higher level of self-efficacy as compared to parents with lower level hope. In lieu of the above results, the second hypothesis is verified. Hence, null hypothesis can be rejected, and hypothesis 2 can be accepted. 
The descriptive statistics of male data revealed that the mean value for the participants' hope scores was 39.10 with a standard deviation of 9.31. Moreover, scores varied between 63 and 25, in the Adult Hope Scale of which the possible minimum score is 8 and the possible maximum score is 64 . On the other hand, statistical results of perceived stress scores, of the overall data showed that the mean was 28.36 and standard deviation was 5.32. The attainable maximum score of Perceived Stress Scale is 40 where the attainable minimum is 0 . The score of perceived stress ranged from 36 to15. The mean and standard deviation for scores of all the participants on self-efficacy were 25.15 and 5.77 respectively and the scores ranged from 39 to 17. The maximum score attainable on the General Self-efficacy Scale was 40 while a minimum of 10 .

The descriptive statistics of the female data revealed that the mean value for the participant's Hope scores was 46.12 with a standard deviation of 10.14 . Moreover, scores varied between 64 and 23, in the Adult Hope Scale of which the possible minimum score is 8and the possible maximum score is 64 . On the other hand, statistical results of Perceived Stress scores, of the overall data showed that the mean was 21.29 and standard deviation was 6.90. The attainable maximum score of Perceived Stress Scale is 40 where the attainable minimum is 0 . The score of perceived stress ranged from 37 to 3 . The mean and standard deviation for scores of all the participants on Self-efficacy were 28.73 and 6.56 respectively and the scores ranged from 40 to 15. The maximum score attainable on the General Self-efficacy Scale was 40 while a minimum of 10. Gender difference in this research was investigated as an ancillary study and because the assumptions of normality not met, the non-parametric Mann Whitney $U$ test was administered.

The obtained $U$ value for participants is 162 . The obtained $\mathrm{Z}$ - score value is -3.6073 . It was observed that the level of significance for perceived stress of participants was 0.003 . Since the obtained value for perceived stress is $<0.05$ level of significance; the data calculated is found to be significant. The results indicated that there is a significant difference in the level of perceived stress between male and female participants. Wherein, males were found to be having higher level of perceived stress than that of females. The obtained $U$ value for participants is 259 . The obtained $\mathrm{Z}$ - score value is 2.0658 . It was observed that the level of significance for perceived stress of participants was 0.0384 . Since the obtained value for selfefficacy is $<0.05$ level of significance, the data calculated is found to be significant. The results indicated that there is a significant difference in the level of self-efficacy between male and female participants. Wherein, female participants were found to be having higher level of self-efficacy than that of male participants.

\section{DISCUSSION}

The first hypothesis was parents of children with intellectual disability having higher level hope will score lower on perceived stress as compared to parents with lower level hope. The statistical result obtained was in line with the hypothesis and also was in line with previous studies. The results obtained of the present study corroborate with the study done to investigate hope in Families of Children with Developmental Disabilities. The focus of this study was to explore and understand the experiences of hope in families of children with disabilities. The result findings revealed hope as a dynamic process that helped parents to reframe their lives in view of the experience of having a child with special needs. Most of the parents in the study reported that accompanying a child with a disability on the road to life became a positive and strengthening experience for them [23].

In a study that researched hope and social support as resilience factors against psychological distress of mothers who cared for children with chronic physical conditions. Subjects were mothers of children who had cerebral palsy, spina bifida, or insulin-dependent diabetes mellitus. The results indicated that both hope and social support were associated negatively and uniquely with distress in these mothers. Perceptions of hope moderated the relationship between disability-related stress and maladjustment, suggesting a buffering effect when stress is high. The current study revealed similar findings where parents having higher level of hope reported lower lever of stress than parents with lower level of hope [24].

A similar research on hope as a psychological resilience factor included mothers \& fathers of children with intellectual disability. It was found that for mothers, regression analyses revealed that lower levels of hope (agency and pathways) and more child behavior problems predicted maternal depression. Positive affect 
was predicted by less problematic child behavior and by higher levels of hope agency. For fathers, anxiety and depression were predicted by low hope agency and positive affect was predicted by high hope agency. Hope agency and pathways interacted in the prediction of maternal depression such that mothers reporting high levels of both hope dimensions reported the lowest levels of depressive symptoms. This study's findings indicate that high hope level in mothers was negatively correlated with depression [25].

A comparative study was done for the purpose to explore the differences on hope and psychological wellbeing among the parents having physically and intellectually disabled children. It also studied if there was any significant relationship between hope and psychological well-being. The results revealed a statistically significant difference between the parents having physically disabled children and parents having intellectually disabled children on hope and psychological well-being variables. The findings also reveal a strong, positive and statistically significant correlation between hope and psychological well-being among the parents having differently abled children [26].

Religion, spirituality and cultural factors also play a crucial role in the perception of the situation as they are a core aspect of global meaning with individuals reappraising their situation as an opportunity. Faith, religious beliefs or spirituality have all been identified as coping mechanisms related to psychological adjustment to stress and this can also provide psychological and emotional support from others [27].

A study was done on behavior problems of children with autism, parental self-efficacy, and mental health. Regression analyses showed that self efficacy mediated the effect of child behavior problems on mothers' anxiety and depression, but there was no evidence that it functioned as a mediator for fathers. However, there was evidence that self-efficacy moderated the effect of child behavior problems on fathers' anxiety. No evidence for the moderating effect of self-efficacy was apparent for mothers [28]. Researchers did a study that examines the contribution of the marital relationship to the well-being of both mothers and fathers of children with developmental disabilities. Results indicated that for both mothers and fathers, greater marital quality predicted lower parenting stress and fewer depressive symptoms above and beyond socio-economic status, child characteristics and social support. In relation to parenting efficacy, marital quality added significant unique variance for mothers but not for fathers. For fathers, greater social support predicted increased parenting efficacy [29].

Compared with mothers of typically developing children, mothers of children with an ASD reported significantly higher fatigue, with overall scores in the moderate range. Factors associated with high levels of fatigue were poor maternal sleep quality, a high need for social support and poor quality of physical activity. Fatigue was also significantly related to other aspects of wellbeing, including stress, anxiety and depression, and lower parenting efficacy and satisfaction. According to the above mentioned past researches; results obtained for the second hypothesis of the current study could be because of the factors such as severity of the child's disruptive and social -emotional behavior, which can also affect the parent's perception of the child's condition and the problems associated with it. Also factors such as marital satisfaction, socio-economic background, social and family support and financial stability may contribute to the results obtained. The gender difference obtained in the current study revealed that males scored lower on hope as compared to the females. It also revealed that males scored higher on perceived stress and lower on self-efficacy than that of females. It indicated that females had better coping mechanisms and psychological well-being as compared to that of males. Various factors could be associated with these results such as socio-economic background, social and family support, accessibility to facilities and internal factors such as locus of control, hardiness, and self-efficacy [30].

Furthermore, research has found gender differences with the women listing family and health-related events more frequently than the men, whereas the men listed relationship, finance and work-related events. The women scored significantly higher than the men on the emotional and avoidance coping styles and lower on rational and detachment coping. The men were found to have more emotional inhibition than the women. And the women scored significantly higher than the men on somatic symptoms and psychological distress [31]. There does appear to be support for stereotypical gender differences in coping strategies. Mother's seeking and expressing emotions with fathers responding stoically playing the supporting role [32]. 


\section{CONCLUSION}

The purpose of the study was to examine whether there is an impact of hope on perceived stress and selfefficacy of parents of children with intellectual disability in Mumbai. The findings indicate that the parents having higher level hope had lower level of perceived stress and higher level of self-efficacy as compared to parents with lower level hope. The results obtained are in line with hypotheses and past research.

\section{REFERENCES}

1. Olsson MB, Hwang CP. Depression in mothers and fathers of children with intellectual disability. J Intellect Disabil Res 2001;45(6):535-43.

2. Watson D. Intraindividual and interindividual analyses of positive and negative affect: their relation to health complaints, perceived stress, and daily activities. J Personal Soc Psychol 1988;54(6):1020-6.

3. Hastings RP, Beck A. Practitioner review: Stress intervention for parents of children with intellectual disabilities. J Child Psychol Psychiatry 2004;45(8):1338-49.

4. Heiman T. Parents of children with disabilities: Resilience, coping, and future expectations. J Dev Phys Disabil 2002;14(2):159-71.

5. Ferguson PM. A place in the family: An historical interpretation of research on parental reactions to having a child with a disability. J Special Educn 2002;36(3):124-31.

6. Bandura A. Self-efficacy: The exercise of control. Macmillan; 1997.

7. Hastings RP, Brown T. Behavior problems of children with autism, parental self-efficacy, and mental health. Am J Ment Retard 2002;107(3):222-32.

8. Kuhn JC, Carter AS. Maternal self- efficacy and associated parenting cognitions among mothers of children with autism. Am J Orthopsychiatry 2006;76(4):564-75.

9. Emerson E. Poverty and children with intellectual disabilities in the world's richer countries. J Intellect Dev Disabil 2004;29(4):319-38.

10. Emerson E. Poverty and people with intellectual disabilities. Dev Disabil Res Rev 2007;13(2):107-13.

11. Stores R, Stores G, Fellows B, Buckley S. Daytime behaviour problems and maternal stress in children with Down's syndrome, their siblings, and non- intellectually disabled and other intellectually disabled peers. Intellect Disabil Res 1998;42(3):228-37.

12. Azeem MW, Dogar IA, Shah S, Cheema MA, Asmat A, Akbar M, Kousar S, Haider II. Anxiety and depression among parents of children with intellectual disability in Pakistan. J Can Acad Child Adolesc Psychiatry 2013;22(4):290-6.

13. Ali A, Hassiotis A, Strydom A, King M. Self stigma in people with intellectual disabilities and courtesy stigma in family carers: A systematic review. Res Dev Disabil 2012;33(6):2122-40.

14. Hastings RP. Parental stress and behaviour problems of children with developmental disability. J Intellect Dev Disabil 2002;27(3):149-60.

15. Khamis V. Psychological distress among parents of children with mental retardation in the United Arab Emirates. Soc Sci Med 2007;64(4):850-7.

16. Gupta RK, Kaur H. Stress among parents of children with intellectual disability. Asia Pacific Disabil Rehab J 2010;21(2):118-26.

17. Hudson AM, Matthews JM, Gavidia- Payne ST, Cameron CA, Mildon RL, Radler GA, Nankervis KL. Evaluation of an intervention system for parents of children with intellectual disability and challenging behaviour. J Intellect Disabil Res 2003;47(4- 5):238-49.

18. Gerstein ED, Crnic K, Blacher J, Baker BL. Resilience and the course of daily parenting stress in families of young children with intellectual disabilities. J Intellect Disabil Res 2009;53(12):981-97.

19. Baker BL, Blacher J, Crnic KA, Edelbrock C. Behavior problems and parenting stress in families of threeyear-old children with and without developmental delays. Am J Ment Retard 2002;107(6):433-44.

20. Snyder CR. The past and possible futures of hope. J Soc Clin Psychol 2000;19(1):11-28.

21. Cohen S, Kamarck T, Mermelstein R. Perceived stress scale. Measuring stress: A guide for health and social scientists. 1994.

22. Chen G, Gully SM, Eden D. Validation of a new general self-efficacy scale. Organiz Res Methods 2001;4(1):62-83.

23. Kausar S, Jevne RF, Sobsey D. Hope in families of children with developmental disabilities. J Dev Disabil 2003;10(1):35-46. 
24. Sawyer MG, Bittman M, La Greca AM, Crettenden AD, Harchak TF, Martin J. Time demands of caring for children with autism: what are the implications for maternal mental health?. J Autism Dev Disord 2010;40(5):620-8.

25. Lloyd TJ, Hastings R. Hope as a psychological resilience factor in mothers and fathers of children with intellectual disabilities. J Intellect Disabil Res 2009;53(12):957-68.

26. Basgül SS, Üneri ÖS, Çakin-Memik N. Parents' perception of the quality of life of children with intellectual disabilities. Turkish J Pediatr 2011;53(5):541-8.

27. Edwardraj S, Mumtaj K, Prasad JH, Kuruvilla A, Jacob KS. Perceptions about intellectual disability: a qualitative study from Vellore, South India. J Intellect Dis Res 2010;54(8):736-48.

28. MacDonald EE, Hastings RP, Fitzsimons E. Psychological acceptance mediates the impact of the behaviour problems of children with intellectual disability on fathers' psychological adjustment. J Appl Res Intellect Disabil 2010;23(1):27-37.

29. Cohen SR. Advocacy for the "Abandonados": Harnessing cultural beliefs for Latino families and their children with intellectual disabilities. J Policy Pract Intellect Disabil 2013;10(1):71-8.

\author{
$* * * * * * * * * * * * * * * * * * * * * *$ \\ Acknowledgements - Nil \\ Source of Funding - Nil \\ Conflict of Interest - Nil
}

